\title{
Clinicopathological and Endoscopic Features of Raspberry-Shaped Gastric Cancer in Helicobacter pylori-Uninfected Patients
}

\author{
Noboru Yatagai ${ }^{a}$ Hiroya Ueyama ${ }^{a}$ Muneo Ikemura ${ }^{a}$ Ryota Uchida ${ }^{a}$ \\ Hisanori Utsunomiya ${ }^{a}$ Daiki Abe ${ }^{a}$ Shotaro Oki ${ }^{a}$ Nobuyuki Suzukia \\ Atsushi lkeda ${ }^{a}$ Yoichi Akazawa $^{a}$ Hiroyuki Komoria ${ }^{a}$ Tsutomu Takeda ${ }^{a}$ \\ Kohei Matsumoto $^{a}$ Kumiko Ueda $^{a}$ Kenshi Matsumoto ${ }^{a}$ Daisuke Asaoka $^{a}$ \\ Mariko Hojo ${ }^{a}$ Sho Tsuyama $^{b}$ Takuo Hayashi $^{b}$ Takashi Yao $^{c}$ \\ Akihito Nagahara ${ }^{a}$
}

${ }^{a}$ Department of Gastroenterology, Juntendo University School of Medicine, Tokyo, Japan; 'Department of Human Pathology, Juntendo University School of Medicine, Tokyo, Japan; 'Department of Human Pathology, Juntendo University Graduate School of Medicine, Tokyo, Japan

\section{Keywords}

Raspberry-shaped gastric cancer · Gastric adenocarcinoma of foveolar-type · Helicobacter pylori-uninfected gastric cancer - Gastric adenocarcinoma of fundic gland type. Gastric adenocarcinoma of fundic gland mucosa type

\begin{abstract}
Background: Gastric adenocarcinoma of foveolar type (GA$\mathrm{FV}$ ) is a raspberry-shaped gastric cancer (RSGC) and garners much attention as $\mathrm{H}$. pylori (Hp)-uninfected gastric cancer. However, the classification and clinicopathological and endoscopic features of RSGCs in Hp-uninfected patients are poorly defined. We designed a new histopathological classification of RSGC and compared them via endoscopic and clinicopathological characteristics. Summary: From 996 patients with early gastric cancers resected by endoscopy in our hospital, we studied 24 RSGC lesions from 21 (2.4\%) $\mathrm{Hp}$ uninfected patients. RSGCs were classified into 3 histological types as follows: GA-FV $(n=19)$, gastric adenocarcinoma of fundic gland type (GA-FG, $n=2)$, and gastric adenocarcinoma of fundic gland mucosa type (GA-FGM, $n=3)$. Most of the
\end{abstract}

lesions were found at the greater curvature of the upper or middle third of the stomach. GA-FV lesions were homogeneously reddish and frequently accompanied with a whitish area around the tumor and an irregular microvascular (MV) pattern; these features were confirmed histopathologically by the presence of homogeneous neoplastic foveolar epithelium with foveolar hyperplasia around the tumors. GA-FG lesions might be heterogeneously reddish with a submucosal tumor shape and regular MV pattern; these were confirmed by the presence of covered or mixed nonneoplastic epithelium on deeper regions of tumors. GA-FGM lesions might be homogeneously reddish and occasionally had a submucosal tumor shape and irregular MV pattern; these were confirmed by the presence of homogeneous neoplastic foveolar epithelium on deeper regions of the tumors. Key Messages: RSGCs in Hp-uninfected patients are classified into 3 histopathological types. For accurate diagnosis of RSGCs, it may be necessary to fully understand endoscopic features of these lesions based on these histological characteristics and to take a precise biopsy. @ 2020 The Author(s)

Published by S. Karger AG, Basel karger@karger.com www.karger.com/dig

Karger ${ }^{\prime \prime} \div$

GOPEN ACCESS
(C) 2020 The Authos(s)

Published by S. Karger AG, Basel

This is an Open Access article licensed under the Creative Commons Attribution-NonCommercial-4.0 International License (CC BY-NC) (http://www.karger.com/Services/OpenAccessLicense), applicable to the online version of the article only. Usage and distribution for commercial purposes requires written permission.
Hiroya Ueyama

Department of Gastroenterology, Juntendo University School of Medicine

2-1-1 Hongo, Bunkyo-Ku

Tokyo 113-8421 (Japan)

psyro@juntendo.ac.jp 


\section{Introduction}

The development of gastric cancer (GC) is associated with histological changes such as atrophic gastritis and intestinal metaplasia due to infection with $\mathrm{H}$. pylori $(\mathrm{Hp})$ $[1,2]$. In Japan, the majority of patients with GC are $\mathrm{Hp}$ positive, $H p$-eradication, and $H p$-uninfected GC accounts for $<1 \%[3,4]$. However, as eradication therapy of $\mathrm{Hp}$ has been performed widely, the proportion of $\mathrm{Hp}$ positive patients has gradually decreased, and $\mathrm{Hp}$-uninfected GC is expected to increase relatively [5].

Previous studies have classified $\mathrm{Hp}$-uninfected GCs into 4 major histopathological types: signet ring cell carcinomas $[6,7]$, gastric adenocarcinoma of fundic gland (GA-FG) type $[8,9]$, gastric adenocarcinoma of fundic gland mucosa (GA-FGM) type $[10,11]$, and differentiated type GC with low-glade atypia [12]. Recently, gastric adenocarcinoma of foveolar-type (GA-FV), which is diagnosed as a differentiated type GC with low-glade atypia in $\mathrm{Hp}$-uninfected GCs, has newly been categorized as a subtype of raspberry-shaped gastric cancer (RSGC) [13, 14]. We recognize GA-FV as a well-differentiated adenocarcinoma based on the Japanese Classification of Gastric Carcinoma (JCGC), although it has been described as a foveolar-type adenoma by WHO classification $[14,15]$. On the contrary, we have observed some cases of GA-FG and GA-FGM that presented with reddish protruded lesions similar to those observed in RSGC [16]. The classification and clinicopathological and endoscopic features of RSGC subtypes have not been well elucidated. Therefore, we aimed to establish a new histopathological classification of RSGC and clarify the clinicopathological and endoscopic features of RSGCs in $\mathrm{Hp}$-uninfected patients.

\section{Materials and Methods}

\section{Outcomes}

We collected RSGC lesions in $\mathrm{Hp}$-uninfected patients from 996 early GC resections performed by endoscopy in Juntendo University Hospital, Tokyo, Japan, between April 2009 and December 2019. We designed a new histopathological classification of RSGC and compared them via endoscopic and clinicopathological evaluation. Follow-up periods were defined as the survival time from treatment to last outpatient visit or annual endoscopy.

\section{Hp Infection Status}

$\mathrm{Hp}$-uninfected patients were defined as cases satisfying the following 4 criteria: (1) endoscopic findings showed no gastric mucosal atrophy or C-1 according to the Kimura-Takemoto Classification [17]; (2) pathological findings showed no gastric atrophy of the resected specimen; (3) clinical findings were negative for at least 1 of the 3 following tests: ${ }^{13} \mathrm{C}$-urea breath test (UBT; cutoff value $<2.5 \%$, Otsuka, Tokushima, Japan), the $H p$ stool antigen test (Premier Platinum HpSA; Meridian, Cincinnati, OH, USA), or the absence of serum immunoglobulin $\mathrm{G}$ antibodies against $H p$ ( $\mathrm{HpAb}$; cutoff value $<3 \mathrm{U} / \mathrm{mL}$, Eiken, Tokyo, Japan); (4) no history of $H p$-eradication therapy $[17,18]$.

\section{Histopathological and Immunohistochemical Assessment}

Resected specimens were formalin fixed, sectioned, and subjected to hematoxylin and eosin staining and immunohistochemistry. Monoclonal antibodies against the following markers were used: MUC5AC (Novocastra, Newcastle upon Tyne, UK) as a marker for gastric foveolar cells, MUC6 (Novocastra, Newcastle upon Tyne, UK) as a marker for gastric mucous neck cells and pyloric glands, MUC2 (Leica, Newcastle upon Tyne, UK) as a marker for intestinal goblet cells, CD10 (Novocastra, Newcastle upon Tyne, UK) as a marker for the small intestinal brush border, pepsinogen I (Abcam, Cambridge, UK) as a marker for chief cells, $\mathrm{H}^{+}$/ $\mathrm{K}^{+}$-ATPase a subunit (MBL, Nagoya, Japan) as a marker for parietal cells, Ki-67 (MIB-1, DAKO, Glostrup, Denmark), and p53 (BioGenex, CA, USA). When $>10 \%$ of the area exhibited staining, a sample was classified as positive. The Ki-67 labeling index (LI) was defined as the percentage of MIB-1-positive nuclei. The Ki-67 LI was determined by counting at least 1,000 nuclei in the selected fields at $\times 400$ magnification. Samples were only scored as p53positive when distinct nuclear staining was diffusely recognized across the tumor area.

The definitions used for pathological diagnosis were as follows. GA-FV was defined as differentiated adenocarcinoma which was composed of cells that resemble foveolar epithelium and was positive for MUC5AC. GA-FG was defined as a differentiated adenocarcinoma which was composed of cells that resemble fundic glands and was positive for pepsinogen $\mathrm{I}$ or $\mathrm{H}^{+} / \mathrm{K}^{+}$-ATPase. GAFGM was defined as a differentiated adenocarcinoma composed of cells that resemble foveolar epithelium and fundic glands. Neoplastic foveolar epithelium had to be MUC5AC-positive and neoplastic fundic gland cells had to be positive for pepsinogen $\mathrm{I}_{\text {or }} \mathrm{H}^{+}$/ $\mathrm{K}^{+}$-ATPase. Characteristics considered during histopathological analysis included the presence or absence of foveolar hyperplasia around the tumors (Fig. 1f) and an association with covered or mixed nonneoplastic epithelium (Fig. 2d). Histopathological diagnoses were made by a pathologist specialized in gastrointestinal pathology (T.Y.) at our hospital.

\section{Endoscopic Assessment}

A raspberry-like reddish protruded lesion with white-light endoscopy (WLE) was diagnosed as RSGC (Fig. 1). Endoscopic findings with WLE included the presence or absence of nodular/granular structures, redness (homogeneous/heterogeneous), submucosal tumor shape, whitish area around the tumor, and coexistence with white and flat elevated lesions and fundic gland polyps. Magnifying endoscopy with NBI (ME-NBI) was performed for 24 lesions, and we employed a "vessel plus surface" classification system [19] which is the main algorithm of magnifying endoscopy simple diagnostic algorithm for early gastric cancer (MESDA-G) [20] for a ME-NBI diagnosis of EGC. Analysis of endoscopic findings with ME-NBI considered the presence or absence of demarcation line, microvascular (MV) pattern, microsurface (MS) pattern, irregular inner edge shape of marginal crypt epithelium (MCE) (Fig. 1c, ar- 

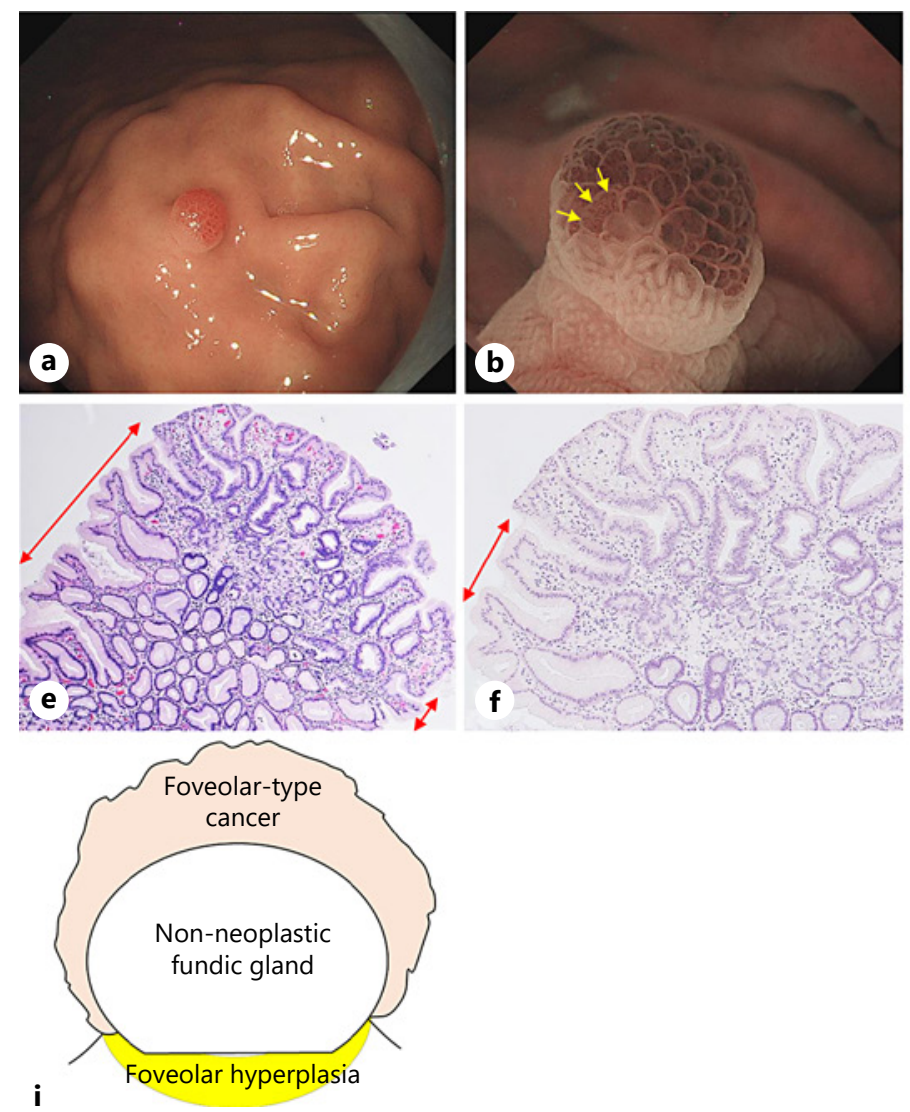

Fig. 1. GA-FV. a WLE revealed a reddish superficial elevated lesion with a whitish area around the tumor, approximately $3 \mathrm{~mm}$ in size, on the greater curvature of the upper third of the stomach. Magnifying endoscopy with narrow-band imaging showed an irregular MV pattern plus a regular MS pattern with a demarcation line and an irregular inner edge shape of MCE (arrow, b). Irregular inner edge shape of MCE (arrow) and irregular outer edge shape of MCE (arrowhead) shown in c. The caliber change of MCE (arrow) is shown in d. Histological features of the CFP specimen showed

row), irregular outer edge shape of MCE (Fig. 1c, arrowhead), and caliber change of MCE (Fig. 1d, arrowhead). Two expert endoscopists (H.U. and N.Y.) retrospectively evaluated all the WLE and ME-NBI images. If their diagnoses differed, a final diagnosis was reached following discussion between these experts.

\section{Results}

Based on the new histopathological classification of RSGCs, RSGC lesions $(n=24)$ were subclassified into GAFV subtypes ( $n=19$, Fig. 1$)$, GA-FG subtypes figure $(n=$ 2, Fig. $2 \mathrm{a}-\mathrm{j})$, and GA-FGM subtypes $(n=3$, Fig. $2 \mathrm{k}-\mathrm{t})$.

Raspberry-Shaped Gastric Cancers in $H$. pylori-Uninfected Patients
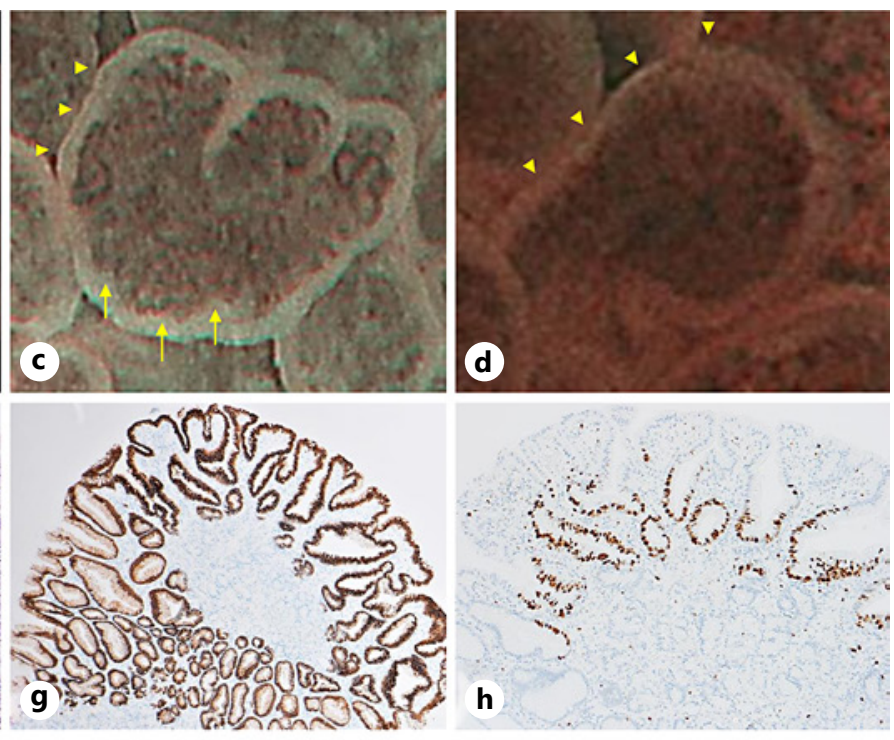

well-differentiated adenocarcinoma mimicking foveolar epithelium in the superficial layer. Foveolar hyperplasia was present in the superficial layer around the tumor (e, f, double arrow). The lesion was positive for MUC5AC (g) and Ki-67 was overexpressed (LI $50 \%)(\mathbf{h})$. The structural schema of GA-FV is shown in i. Original magnification: $\times 40(\mathbf{e}, \mathbf{g}), \times 100(\mathbf{f}, \mathbf{h})$. GA-FV, gastric adenocarcinoma of foveolar-type; WLE, white-light endoscopy; LI, labeling index; MV, microvascular; MS, microsurface; MCE, marginal crypt epithelium; CFP, cold forceps polypectomy.

\section{Clinicopathological Features of RSGCs}

The clinicopathological features of RSGCs are summarized in Table 1. Twenty-four RSGC lesions (2.4\%) from $21 \mathrm{Hp}$-uninfected patients (14 males and 7 females) which consisted of 20 solitary cancers in 20 patients and 4 lesions of multiple synchronous cancers in 1 patient were studied. In all RSGC lesions, tumors were located at the upper third/middle third/lower third of the stomach in $18 / 6 / 0$ of the RSGC cases, respectively, and at the greater curvature/lesser curvature/anterior wall/posterior wall in 20/1/0/3 cases, respectively. The therapy method was biopsy/cold forceps polypectomy (CFP)/endoscopic mucosal resection/endoscopic submucosal dissection in 4/8/7/5 cases, respectively. Small GA-FV le- 

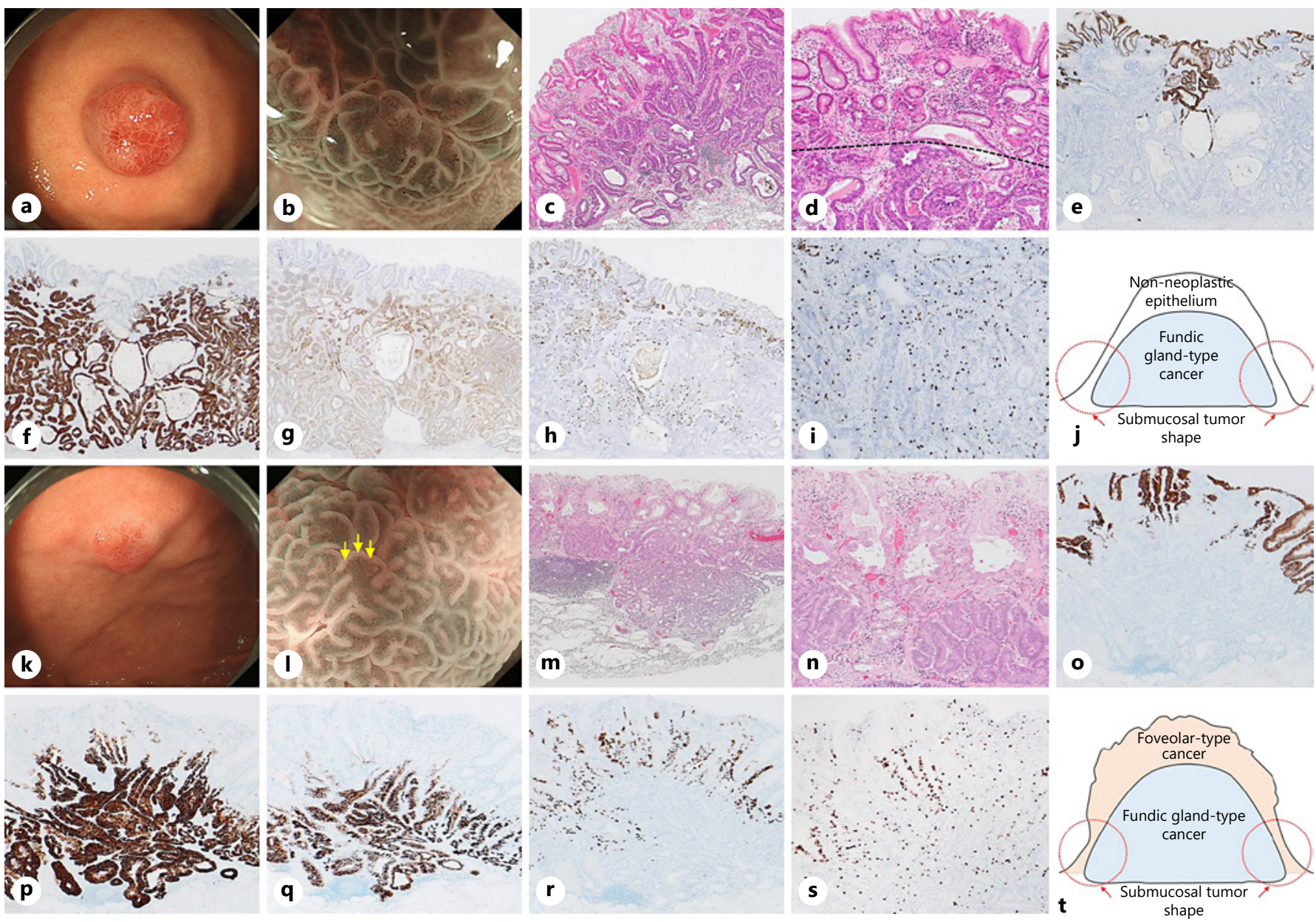

Fig. 2. GA-FG (a-j). a WLE revealed a heterogeneous reddish protruded lesion with submucosal tumor shape, approximately 12 $\mathrm{mm}$ in size, on the greater curvature of the upper third of the stomach. b Magnifying endoscopy with narrow-band imaging showed a regular MV pattern plus a regular MS pattern with a demarcation line. c Histological features of the endoscopic submucosal dissection specimen showed well-differentiated adenocarcinoma mimicking gastric fundic glands with an irregular branching structure in the deep layer of the lamina propria mucosa, infiltrating the submucosal layer $(200 \mu \mathrm{m})$. The superficial layer of the tumor margin was covered with a non-neoplastic epithelium (d, the border between tumor and non-tumor is marked by dotted lines). The lesion was positive for MUC6 (f), pepsinogen-I (g), and $\mathrm{H}^{+} / \mathrm{K}^{+}$ATPase (h) and negative for MUC5AC (e). Ki-67 was overexpressed (LI 20\%) (i). The structural schema of GA-FG is shown in j. GA-FGM (k-t). WLE revealed a reddish superficial elevated lesion with submucosal tumor shape, approximately $15 \mathrm{~mm}$ in size, on the posterior wall of the upper third of the stomach (k). Magnifying endoscopy with narrow-band imaging showed the absence of a MV pattern plus a regular MS pattern with a demarcation line and an irregular inner edge shape of MCE (arrow, I). Histological features of the endoscopic submucosal dissection specimen showed well-differentiated adenocarcinoma mimicking foveolar epithelium in the superficial layer and mimicking gastric fundic glands with irregular branching structure in the deep layer of the lamina propria mucosa, infiltrating the submucosal layer $(700 \mu \mathrm{m})$ $(\mathbf{m}, \mathbf{n})$. The lesion was positive for MUC5AC (o), MUC6 (p), pepsinogen $\mathrm{I}(\mathbf{q})$, and $\mathrm{H}^{+} / \mathrm{K}^{+}$-ATPase $(\mathbf{r})$. Ki-67 was overexpressed (LI $10 \%)(\mathbf{s})$. The structural schema of GA-FGM is shown in $\mathbf{t}$. Original magnification: $(\mathbf{c}, \mathbf{e}, \mathbf{f}, \mathbf{g}, \mathbf{h}, \mathbf{m}, \mathbf{o}, \mathbf{p}, \mathbf{q}, \mathbf{r}) \times 40,(\mathbf{d}, \mathbf{i}, \mathbf{n}, \mathbf{s}) \times 100$. GA-FG, gastric adenocarcinoma of fundic gland type; $\mathrm{MV}$, microvascular; MS, microsurface; LI, labeling index; GA-FGM, gastric adenocarcinoma of fundic gland mucosa type; WLE, white-light endoscopy; MCE, marginal crypt epithelium. sions $<5 \mathrm{~mm}$ were completely resected by biopsy or CFP. No remnant lesions were detected among 12 GA-FV cases after biopsy or CFP during the follow-up period. Lymphovascular invasion and lateral and vertical margins were not assessed in the lesions resected by biopsy and CFP; however, the other RSGC lesions were negative for these features. 
Table 1. Clinicopathological features of RSGCs

\begin{tabular}{|c|c|c|c|}
\hline & GA-FV & GA-FG & GA-FGM \\
\hline Patients & $n=16$ & $n=2$ & $n=3$ \\
\hline Age (mean), years & 54.4 & 65.5 & 48.7 \\
\hline Gender (male/female) & $12 / 4$ & $0 / 2$ & $2 / 1$ \\
\hline$H p$ infection status (uninfected/positive/post-eradicated) & $16 / 0 / 0$ & $2 / 0 / 0$ & $3 / 0 / 0$ \\
\hline PPI administration, $\%$ & 31.3 & 0 & 0 \\
\hline Range & $2-120$ & $44-51$ & $1-7$ \\
\hline Outcome & All alive NED & All alive NED & All alive NED \\
\hline Lesions & $n=19$ & $n=2$ & $n=3$ \\
\hline Tumor location, U/M/L & $13 / 6 / 0$ & $2 / 0 / 0$ & $3 / 0 / 0$ \\
\hline (GC/LC/ant/post) & $17 / 1 / 0 / 1$ & $2 / 0 / 0 / 0$ & $1 / 0 / 0 / 2$ \\
\hline Tumor size (mean), mm & 3.0 & 8.5 & 8.0 \\
\hline Invasion depth (M/SM) & $19 / 0$ & $0 / 2$ & $1 / 2$ \\
\hline Distance of SM invasion (mean) (range) & NA & $250 \mu \mathrm{m}(200-300 \mu \mathrm{m})$ & $500 \mu \mathrm{m}(300-700 \mu \mathrm{m})$ \\
\hline Lymphatic invasion $(+)$ & $0 \%(0 / 7, \mathrm{NA}: 12)$ & $0 \%$ & $0 \%$ \\
\hline Venous invasion $(+)$ & $0 \%(0 / 7, \mathrm{NA}: 12)$ & $0 \%$ & $0 \%$ \\
\hline Lateral margin $(+)$ & $0 \%(0 / 6, \mathrm{NA}: 13)$ & $0 \%$ & $0 \%$ \\
\hline Vertical margin $(+)$ & $0 \%(0 / 6$, NA: 13$)$ & $0 \%$ & $0 \%$ \\
\hline Foveolar hyperplasia around tumor & $94.1 \%(16 / 17, \mathrm{NA}: 2)$ & $0 \%$ & $66.7 \%$ \\
\hline Covered or mixed nonneoplastic epithelium & $23.5 \%(4 / 17, \mathrm{NA}: 2)$ & $100 \%$ & $0 \%$ \\
\hline
\end{tabular}

GA-FV, gastric adenocarcinoma of foveolar-type; GA-FG, gastric adenocarcinoma of fundic gland type; GA-FGM, gastric adenocarcinoma of fundic gland mucosa type; RSGCs, raspberry-shaped gastric cancers; $\mathrm{H} p$; Helicobacter pylori; PPI, proton pump inhibitor; U, upper third; M, middle third; L, lower third; GC, greater curvature; LC, lesser curvature; Ant, anterior wall; Post, posterior wall; 0-I, protruded; 0-IIa, superficial elevated; CFP, cold forceps polypectomy; EMR, endoscopic mucosal resection; ESD, endoscopic submucosal dissection; tub1, well-differentiated tubular adenocarcinoma; pap, papillary adenocarcinoma; M, mucosal layer; SM, submucosal layer; NA, not assessed; NED, no evidence of disease.

Histopathological Features and Immunohistochemical Analysis of RSGCs

The histopathological features of RSGCs are summarized in Table 1. Immunohistochemically, all GA-FV lesions were MUC5AC-positive and negative for MUC6, MUC2, CD10, pepsinogen $\mathrm{I}$, and $\mathrm{H}^{+} / \mathrm{K}^{+}$-ATPase. All GAFG lesions were positive for MUC6, pepsinogen I and negative for MUC5AC, MUC2, and CD10. All GA-FGM lesions were positive for MUC5AC, MUC6, and pepsinogen I and negative for MUC2 and CD10. Some lesions in GA-FG and GA-FGM were focally positive for $\mathrm{H}^{+} / \mathrm{K}^{+}$ATPase. All RSGC lesions were classified into gastric mucin phenotypes. The mean Ki-67 LIs of GA-FV and GAFGM were relatively higher than GA-FG (GA-FV/GAFG/GA-FGM: 44.1/15/30\%). No RSGC lesions exhibited p53 overexpression.

Raspberry-Shaped Gastric Cancers in $H$. pylori-Uninfected Patients
Endoscopic Features of RSGCs Based on These Histological Characteristics

The endoscopic features of RSGCs in WLE and MENBI are summarized in Table 2. GA-FV were characterized by homogeneous reddish lesions and frequently accompanied with whitish area around the tumor in WLE and an irregular MV pattern and irregular inner edge shape of MCE in ME-NBI; these were confirmed histopathologically by the presence of homogeneous neoplastic foveolar epithelium with foveolar hyperplasia around the tumors (Fig. 1i). Although the clinicopathological and endoscopic features of GA-FG and GA-FGM are not clear because of a small number of cases, our findings were as follows. WLE revealed that GA-FG had heterogeneous reddish lesions and submucosal tumor shape. MENBI indicated that these were accompanied by a regular MV pattern; these were confirmed histopathologically by 
Table 2. Endoscopic features of RSGCs

$\begin{array}{lll}\text { GA-FV } & \text { GA-FG } & \text { GA-FGM } \\ (n=19) & (n=2) & (n=3)\end{array}$

$\begin{array}{llll}\text { WLE } & & & \\ \text { Nodular/granular structure } & 84.2 \% & 100 \% & 33.3 \% \\ \text { Reddish color (homogeneous/heterogeneous) } & 19 / 0 & 0 / 2 & 3 / 0 \\ \text { Submucosal tumor shape } & 0 \% & 100 \% & 33.3 \% \\ \text { Whitish area around tumor } & 57.9 \% & 0 \% & 0 \% \\ \text { Coexistence with white and flat elevated lesion } & 15.3 \% & 0 \% & 0 \% \\ \text { Coexistence with fundic gland polyp } & 78.9 \% & 100 \% & 33.3 \% \\ \text { Magnifying endoscopy with narrow-band imaging } & n=15 & n=2 & n=3 \\ \text { MESDA-G (cancer/non-cancer) } & 11 / 4 & 1 / 1 & 2 / 1 \\ \text { Demarcation line } & 100 \% & 100 \% & 100 \% \\ \text { MV pattern (regular/irregular/absent) } & 0 / 11 / 4 \text { (low confidence } 2) & 1 / 1 / 0 & 0 / 2 / 1 \\ \text { MS pattern (regular/irregular/absent) } & 15 / 0 / 0 & 2 / 0 / 0 & 3 / 0 / 0 \\ \text { Irregular inner edge shape of MCE } & 73.3 \% & 50 \% & 66.7 \% \\ \text { Irregular outer edge shape of MCE } & 40 \% & 0 \% & 0 \% \\ \text { Caliber change of MCE } & 33.3 \% & 0 \% & 0 \%\end{array}$

GA-FV, gastric adenocarcinoma of foveolar-type; GA-FG, gastric adenocarcinoma of fundic gland type; GAFGM, gastric adenocarcinoma of fundic gland mucosa type; RSGCs, raspberry-shaped gastric cancers; MCE, marginal crypt epithelium; MESDA-G, magnifying endoscopy simple diagnostic algorithm for early gastric cancer; MV, microvascular; MS, microsurface; WLE, white-light endoscopy.

the presence of covered or mixed nonneoplastic epithelium on tumor mass in the deep areas of the neoplasm (Fig. 2j). WLE revealed that GA-FGM had homogeneous reddish lesions and the occasional presence of a submucosal tumor shape while ME-NBI indicated an irregular MV pattern and irregular inner edge shape of MCE; these were confirmed histopathologically by the presence of homogeneous neoplastic foveolar epithelium and tumor mass in the deep areas of the neoplasm (Fig. 2t). ME-NBI was performed in 20 lesions (GA-FV/GA-FG/GA-FGM: $15 / 2 / 3$ ). The diagnostic accuracy of ME-NBI using MESDA-G was all/GA-FV/GA-FG/GA-FGM: 70/86.7/50/ $66.7 \%$.

\section{Discussion}

Our frequency of RSGCs in $\mathrm{Hp}$-uninfected patients (2.4\%) is higher than that of $H p$-uninfected GC in previous reports $[3,4]$. We speculate that RSGCs might have been overlooked during past endoscopic evaluations, since they resemble hyperplastic polyps, reddish fundic gland polyps, or PPI-related polyps [21]. Thus, endoscopists may not have recognized that some reddish protruded lesion should have been diagnosed as RSGCs. Our current observations therefore stress this importance of close collaboration between endoscopists and pathologists in order to provide an accurate diagnosis of RSGCs. In upper gastrointestinal endoscopy, when a bright reddish protruded lesion is observed in the greater curvature of the upper or middle third of the stomach in Hp-uninfected patients, RSGC should be suspected. Careful observation of color uniformity, tumor morphology, whitish area around the tumor, and the findings of ME-NBI is required to avoid overlooking RSGC, since these tumors are relatively small and the differentiation from raspberryshaped benign lesions is challenging. Currently, a biopsy is required for accurate diagnosis of RSGCs when careful observation with WLE and ME-NBI is not feasible.

As for ME-NBI in GA-FV, all lesions which MV pattern could be visualized was diagnosed as cancer by irregular MV pattern, similar to previous reports [14]. The judgment of MV irregularity may be useful for accurate diagnosis of RSGCs, since the MS pattern did not contribute to diagnosis of malignancy by ME-NBI. Although the MS in GA-FV had a roughly square morphology, it was homogeneous, distributed symmetrically, and arranged regularly; it was therefore diagnosed as regular in all lesions. Although an irregular inner edge shape of MCE may be useful for diagnosis of cancer, an irregular outer edge shape of MCE and caliber change of MCE were not useful. We speculate that the irregularity of inner edge of 
MCE was due to deformation of the MV outer edge (Fig. 1c). Therefore, identification of the MV pattern is especially important for the accurate diagnosis of RSGC.

GA-FV can be diagnosed endoscopically as mentioned above; however, GA-FG and GA-FGM cannot be clearly characterized due to a small number of cases in this study. Endoscopically, the most common features of GA-FG were submucosal tumor shape, whitish color, dilated vessels with branching architecture, and background mucosa without atrophic change [9]. GA-FGM has been reported to be frequently diagnosed as irregular MV/MS pattern in ME-NBI due to the exposure of neoplastic foveolar epithelium on the surface $[10,16]$. Raspberryshaped GA-FG and GA-FGM were extremely rare, and these endoscopic features were not fully elucidated in previous reports [16]. In this study, raspberry-shaped GA-FG had submucosal tumor shape and raspberryshaped GA-FGM had irregular MV pattern in ME-NBI, which were partially similar to the typical type of GA-FG and GA-FGM. However, further studies will be needed to characterize the raspberry-shaped GA-FG and GA-FGM and to discriminate them from GA-FV.

Immunohistochemical staining showed a pure gastric mucin phenotype in all RSGCs, and Ki-67 LI was relatively high in GA-FV. Although a pure gastric mucin phenotype and highly Ki-67 LI are usually associated with poor prognosis in GC $[22,23]$, GA-FVs did not show invasive and metastatic behavior. In addition, the GA-FG and GA-FGM in our study tended to infiltrate the submucosal layer as previously reported [16]. This was, in spite of their small diameter, low cellular atypia, no lymphovascular invasion, low proliferative activity, and a lack of p53 protein overexpression. There is a discrepancy between the clinical and histopathological features in each type of RSGCs. However, GA-FGM should be dealt with separately, since some cases with high cellular atypia have been reported as aggressive GA-FGM variants with high malignant potential $[24,25]$. Evaluation of the natural history and malignant potential of each RSGC subtype will be needed in the future.

This study has some limitations. First, it is a retrospective observational analysis performed at a single center with a small sample size. Second, we could not correct for and analyze raspberry-shaped benign lesions. Further investigation will be required to establish an algorithm for endoscopic diagnosis of RSGC that incorporates their separation from raspberry-shaped benign lesions.

In conclusion, histology allows the classification of RSGCs in $\mathrm{Hp}$-uninfected patients into GA-FV, GA-FG, and GA-FGM subtypes, and RSGCs may be diagnosed cor- rectly based on color uniformity, tumor morphology, and presence of whitish area around the tumor with WLE; ME-NBI may provide further discrimination based on the presence or absence of an irregular MV pattern and an irregular inner edge shape of MCE. Although all endoscopic features in each type were explained adequately by the specific histopathological findings of each type, endoscopic discrimination of each type might be still difficult. Therefore, these endoscopic and clinicopathological features should be taken into consideration to diagnose RSGC correctly by pathological examination of biopsy specimens.

\section{Acknowledgements}

We thank Isao Kurahayashi, Noriko Sasahara, and Satomi Saito (Department of Human Pathology, Juntendo University School of Medicine) for their excellent technical assistance.

\section{Statement of Ethics}

This study was reviewed and approved by the Juntendo University School of Medicine Institutional Review Board (\#2019050). Patient consent was not required because the analysis used anonymous clinical data obtained after each patient had already given verbal and documental consent for treatment. It is not possible to identify an individual from the data presented.

\section{Conflict of Interest Statement}

The authors have no conflicts of interest to declare.

\section{Funding Sources}

The authors did not receive any funding.

\section{References}

1 Schistosomes, liver flukes and Helicobacter pylori. IARC working group on the evaluation of carcinogenic risks to humans. Lyon, 7-14 June 1994. IARC Monogr Eval Carcinog Risks Hum; 1994. Vol. 61; 1-241.

2 Uemura N, Okamoto S, Yamamoto S, Matsumura N, Yamaguchi S, Yamakido M, et al. Helicobacter pylori infection and the development of gastric cancer. N Engl J Med. 2001 Sep 13;345(11):784-9.

3 Matsuo T, Ito M, Takata S, Tanaka S, Yoshihara M, Chayama K. Low prevalence of Helicobacter pylori-negative gastric cancer among Japanese. Helicobacter. 2011 Dec; 16(6):415-9. 
4 Ono S, Kato M, Suzuki M, Ishigaki S, Takahashi M, Haneda M, et al. Frequency of Helicobacter pylori-negative gastric cancer and gastric mucosal atrophy in a Japanese endoscopic submucosal dissection series including histological, endoscopic and serological atrophy. Digestion. 2012;86(1):59-65.

5 Yamada A, Kaise M, Inoshita N, Toba T, Nomura K, Kuribayashi Y, et al. Characterization of Helicobacter pylori-naïve early gastric cancers. Digestion. 2018;98(2):127-34.

6 Yamamoto Y, Fujisaki J, Omae M, Hirasawa T, Igarashi M. Helicobacter pylori-negative gastric cancer: characteristics and endoscopic findings. Dig Endosc. 2015 Jul;27(5):551-61.

7 Kiso M, Yoshihara M, Ito M, Inoue K, Kato K, Nakajima S, et al. Characteristics of gastric cancer in negative test of serum anti-Helicobacter pylori antibody and pepsinogen test: a multicenter study. Gastric Cancer. 2017 Sep; 20(5):764-71.

8 Ueyama H, Yao T, Nakashima Y, Hirakawa K, Oshiro Y, Hirahashi M, et al. Gastric adenocarcinoma of fundic gland type (chief cell predominant type): proposal for a new entity of gastric adenocarcinoma. Am J Surg Pathol. 2010 May;34(5):609-19.

9 Ueyama H, Matsumoto K, Nagahara A, Hayashi T, Yao T, Watanabe S. Gastric adenocarcinoma of the fundic gland type (chief cell predominant type). Endoscopy. $2014 \mathrm{Feb}$; 46(2):153-7.

10 Fujiwara S, Yao K, Imamura K, Tanabe H, Yasaka T, Ishihara $\mathrm{Y}$, et al. M-NBI findings of gastric adenocarcinoma of fundic gland type and adenocarcinoma with differentiation towards the fundic mucosa. Stomach Intest. 2015;50(12):1548-58.
11 Tanabe H, Iwashita A, Ikeda K, Ota A, Yao K. Histopathological characteristics of gastric adenocarcinoma of fundic gland type. Stomach and intestine. Stomach Intest. 2015; 50(12):1469-79.

12 Ueyama H, Matsumoto K, Ikeda A, Yatagai N, Akazawa Y, Komori H, et al. The current situation of Helicobacter pylori-negative (naïve) gastric cancer. Japanese J Helicobacter Res. 2019 Jan;20(2):103-11.

13 Isono Y, Baba Y, Mukai K, Asakawa H, Nose $\mathrm{K}$, Tsuruga $\mathrm{S}$, et al. Gastric adenocarcinoma coexisting with a reddish semipedunculated polyp arising from Helicobacter pylori-negative normal gastric mucosa: a report of two cases. Clin J Gastroenterol. 2018 Dec;11(6): 481-6.

14 Shibagaki K, Fukuyama C, Mikami H, Izumi D, Yamashita N, Mishiro T, et al. Gastric foveolar-type adenomas endoscopically showing a raspberry-like appearance in the Helicobacter pylori-uninfected stomach. Endosc Int Open. 2019 Jun;7(6):E784-E91.

15 Sekine S, Montgomery E, Vieth M. Foveolartype adenoma. WHO classification of digestive system tumours. 5th ed.; 2019. p. 79-80.

16 Ueyama H, Yao T, Nagahara A. Gastric adenocarcinoma of fundic gland type. Stomach Intest. 2018 May;53(5):753-67.

17 Kimura K, Takemoto T. An endoscopic recognition of the atrophic border and its significance in chronic gastritis. Endoscopy. 1969; $1(03): 87-97$.

18 Yagi K, Nakamura A, Sekine A. Characteristic endoscopic and magnified endoscopic findings in the normal stomach without Helicobacter pylori infection. J Gastroenterol Hepatol. 2002 Jan;17(1):39-45.
19 Yao K, Anagnostopoulos GK, Ragunath K. Magnifying endoscopy for diagnosing and delineating early gastric cancer. Endoscopy. 2009 May;41(5):462-7.

20 Muto M, Yao K, Kaise M, Kato M, Uedo N, Yagi K, et al. Magnifying endoscopy simple diagnostic algorithm for early gastric cancer (MESDA-G). Dig Endosc. 2016 May;28(4): 379-93.

21 Miyamoto S, Kato M, Matsuda K, Abiko S, Tsuda M, Mizushima T, et al. Gastric hyperplastic polyps associated with proton pump inhibitor use in a case without a history of $\mathrm{He}$ licobacter pylori infection. Intern Med. 2017; 56(14):1825-9.

22 Koseki K, Takizawa T, Koike M, Ito M, Nihei Z, Sugihara K. Distinction of differentiated type early gastric carcinoma with gastric type mucin expression. Cancer. 2000 Aug 15; 89(4):724-32.

23 Luo G, Hu Y, Zhang Z, Wang P, Luo Z, Lin J, et al. Clinicopathologic significance and prognostic value of $\mathrm{Ki}-67$ expression in patients with gastric cancer: a meta-analysis. Oncotarget. 2017 Jul 25;8(30):50273-83.

24 Ueo T, Yonemasu H, Ishida T. Gastric adenocarcinoma of fundic gland type with unusual behavior. Dig Endosc. 2014 Mar;26(2):293-4.

25 Okumura Y, Takamatsu M, Ohashi M, Yamamoto Y, Yamamoto N, Kawachi H, et al. Gastric adenocarcinoma of fundic gland type with aggressive transformation and lymph node metastasis: a case report. J Gastric Cancer. 2018 Dec;18(4):409-16. 